\title{
Relación entre la gestión de la calidad y el reporte de ocurrencia de eventos adversos en la atención del paciente"
}

\section{Relationship between quality management and the report of adverse events occurring in patient care}

Recibido: 25 de enero de 2012 Revisado: 09 de abril de 2012 Aceptado: 26 de abril de 2012

César Roberto Ocaña Poveda**

Luz Marly Sánche* Bustamante

Convenio Universidad Santo Tomás e ICONTEC

\section{RESUMEN}

El propósito de esta investigación fue el de indagar por la relación que pueda existir entre sistemas de gestión de calidad reconocidos y el reporte de los eventos adversos que ocurren en el interior de las instituciones de salud.

Para el desarrollo del proyecto se contextualizaron las posturas nacionales e internacionales sobre gestión de la calidad y la seguridad del paciente, los cuales se confrontaron con diversos aspectos sobre la realidad que afrontan las instituciones.

Las tres instituciones participantes en el estudio se encuentran ubicadas en Bogotá y cuentan con sistemas de gestión de la calidad reconocidos, que incluyen: acreditación en salud, certificación del Sistema de Gestión de la Calidad y habilitación en salud; siendo esta última utilizada como entidad de control para el estudio.

* Artículo de investigación.

** César Ocaña Poveda. Consultor independiente. Odontólogo. Correo electrónico: ocanace@gmail.com

Luz Marly Sánchez. Profesional externa de ICONTEC. Bacterióloga. Licenciada en Filosofía. Correo electrónico: marlicita25@hotmail.com

Trabajo de Grado para optar al título de Magíster en Calidad y Gestión Integral dirigido por Mg. Sonia Morales. 
Tras la recolección de la información se analizaron los datos y se identificó la relación existente entre los sistemas de gestión de la calidad y el reporte de los eventos adversos, permitiendo determinar su influencia y aporte reflejado en la mejora de la calidad de vida de los usuarios, su apropiación en la institución y la seguridad del paciente, en consonancia con la misión de las instituciones de salud.

Palabras claves: acreditación en salud, certificación de calidad, cultura del reporte, gestión de la calidad.

\section{ABSTRACT}

The purpose of this research was to demonstrate the relationship between quality management systems and reporting recognized adverse events occurring within health institutions.

To develop this project the contextualized national positions and international quality management and patient safety are addressed in various aspects of the reality faced by institutions.

Located in the city of Bogota, the three institutions involved in the study have a system of recognized quality management which includes: health accreditation, certification of the quality management system and health enabling; this last one was used as a control entity for this study.

After collecting the information, it was analyzed and the relationship between the quality systems and the record of the adverse event was identified, allowing the determination of their influence and contribution reflected in improving the quality of life of the users, its appropriation in the institution and patient safety.

Keywords: colombian health care accreditation, culture of reporting, quality management system, quality certification.

\section{INTRODUCCIÓN}

La seguridad del paciente se constituye hoy día como uno de los aspectos esenciales en la gestión de las organizaciones en salud.

El Ministerio de la Protección Social la define como: "El conjunto de elementos estructurales, procesos, instrumentos y metodologías basadas en evidencias científicamente probadas que propenden por minimizar el riesgo de sufrir un evento adverso en el proceso de atención en salud o de mitigar sus consecuencias"1.

Entre los elementos identificables podemos encontrar los lineamientos planteados por el Ministerio de Salud y Protección Social, la políica de seguridad del paciente, protocolos para el manejo de los pacientes, metodologías para la evaluación de los eventos adversos, procesos de detección, metodologías para el análisis y estrategias para profundizar la cultura institucional de seguridad del paciente.

Esto permite determinar que las instituciones han incorporado a su gestión aspectos administrativos relacionados con directrices, recurso humano, infraestructura, procesos, análisis y acciones para la mejora, entre otros, y que son partes esenciales de un sistema de gestión de calidad.

Es de presumir que las IPS acreditadas o certificadas cuentan con mayor conciencia y control hacia la minimización del error, la disminución de no conformidades y el reporte y seguimiento a los eventos adversos. Por lo que es importante identificar si la implementación de estos sistemas impacta de manera positiva a los usuarios al generar beneficios en pro de su seguridad y a la organización en su gestión, en la optimización de procesos con objetivos claros, mecanismos de control y medición como elementos clave de mejoramiento.

1 Ministerio de Salud y Protección Social. Lineamientos para la seguridad del paciente. Bogotá, Colombia, 2007. 


\section{METODOLOGÍA}

La presente investigación tiene un enfoque de preponderancia cualitativa, con su subtipo cualitativo mixto (Cualitativo/Cual-Cuantitativo/Cuan) de ejecución secuencial, de acuerdo con la definición presentada por Hernández Sampieri y Mendoza (2008), donde se recolectan primero los datos cualitativos y cuya intención es explorar el planteamiento con un grupo de participantes en su contexto y posteriormente expandir el entendimiento del problema (Creswell, 2009). Los datos recolectados y analizados en la fase Cuan se utilizan para informar a la otra fase del estudio Cual, para dar respuesta a la pregunta de la investigación.

\section{Recolección de datos}

Los datos obtenidos provienen de las experiencias, procesos y vivencias de los participantes y se recolectan con la finalidad de analizarlos y así responder a las preguntas de la investigación y generar conocimiento.

El desarrollo de la recolección de datos se efectúa en dos momentos:

En el primer momento, se entrega a cada uno de los participantes una encuesta semiestructurada con las temáticas que se abordan en el grupo focal, estas fueron diligenciadas en un tiempo no mayor a 10 minutos. Esta encuesta consta de cinco preguntas relacionadas con el sistema de reporte de incidentes y eventos adversos en la organización, componentes del sistema de reporte, fuentes de información internas y externas para mejorar el reporte, cómo lograr un sistema de reporte exitoso y cómo conseguir un sistema de reporte efectivo. Esta encuesta pretende evidenciar el desarrollo del sistema de reporte en las diferentes instituciones participantes y cómo lo percibe el personal asistencial.
Se dio inicio al grupo focal, donde los investigadores realizaron el debate con las preguntas planteadas en la encuesta semiestructurada y, adicionalmente, surgieron categorías emergentes desde las respuestas dadas por los participantes. El propósito de este instrumento fue recoger la información específica para la parte cualitativa de este trabajo, adicionalmente fue posible encontrar nuevas categorías sobre las percepciones de los participantes dentro de las temáticas planteadas.

Estas entrevistas focales fueron grabadas, pero para mejorar el manejo se hizo la transcripción. Se aplicaron una sola vez en las tres organizaciones participantes del estudio. Se utilizó la grabadora de equipos celulares de alta tecnología, de tal forma que no intimidaran a los participantes y tuviera efectos sobre las respuestas y se volviera un falso subjetivo, es decir, el efecto de un equipo en un entorno por ser nuevo en él.

En el segundo momento, se entregó una encuesta estructurada sobre el reporte de los eventos adversos desde seguridad del paciente y el aporte a los sistemas de la organización, la cual utiliza una escala de actitud tipo Likert, que es una escala ordinal que utiliza una serie de afirmaciones sobre las cuales se obtiene una respuesta del sujeto. Corresponde a un método de escala bipolar que determina los grados positivos, neutrales y negativos de cada enunciado. La escala de Likert, al ser una escala que mide actitudes, es importante que pueda aceptar que las personas tienen actitudes favorables, desfavorables o neutras frente a las situaciones. Al respecto manifiesta Hernández:

Cada elemento o proposición de la escala proporciona una información sobre la actitud de la persona. La acumulación de información y la suma de respuestas permiten decidir la posición que una persona ocupa en el eje hipotético de la actitud. En este sentido se puede afirmar que cada enunciado proporciona una información que no 
permite medir completamente la actitud, pero sí identificar sus tendencias ${ }^{2}$.

La encuesta definitiva se compuso de dos partes: una, en la que se caracterizó la población en sus aspectos generales, tales como: entidad a la que pertenece; cargo que desempeña y tipo de participación como líder. La segunda, son las preguntas tipo afirmación con las que se abordaron a los participantes. El propósito al aplicar esta encuesta fue reconocer cómo se encontraba el sistema de reporte de los eventos adversos en las organizaciones participantes, cómo aportaba a los sistemas de calidad en la organización y servir de insumo para la obtención de información con el fin de profundizar en las categorías definidas.

La aplicación de este instrumento fue suministrada al $100 \%$ de los participantes del grupo focal de la siguiente manera: al terminar el grupo focal se entregó a cada participante para ser diligenciada de acuerdo con la percepción individual. Esta fue aplicada una sola vez.

La información obtenida desde este instrumento aportó al problema de la investigación y a los objetivos específicos dos y tres.

En la tipología de la investigación, la parte cualitativa prima sobre la cuantitativa, el instrumento diseñado para el primer momento recoge las apreciaciones que dan respuesta a la parte cualitativa y la parte cuantitativa se realizó después de la cualitativa, por lo que fue secuencial.

\section{Análisis de datos}

\section{A. Análisis cualitativo}

Para el análisis de los datos cualitativos en la presente investigación se utilizó el Programa de Software

2 HERNÁNDEZ SAMPIERI, Roberto. Metodología de la investigación. Madrid, España: Ed. McGraw-Hill, 2010.
ATLAS.ti, en el que se incluyó la transcripción de las entrevistas realizadas en el grupo focal con el fin de generar el documento primario de cada una de las organizaciones, se codifican las respuestas obtenidas en las tres organizaciones, se establecen las categorías, se identifican las diferentes citas que dan respuestas a las categorías a priori y emergentes identificadas y se obtienen las diferentes familias de acuerdo con las categorías a priori, agrupando las variables identificadas para la posterior interpretación de los datos.

\section{B. Análisis cuantitativo}

Para el análisis cuantitativo se utilizó estadística descriptiva, con un nivel de confianza para esta prueba del $95 \%$, donde la decisión se toma teniendo en cuenta el resultado del valor de $p$, utilizando una hipótesis nula en donde si el valor de $p$ asociado con el resultado observado es menor o igual al nivel de significancia establecido para el estudio, 0,05 nos indica que existe un $5 \%$ de posibilidad de error del valor obtenido. Los datos de las encuestas aplicadas en las tres organizaciones fueron procesados en el software SPSS versión 8.0 (programa computarizado de análisis estadístico). Los resultados obtenidos con el programa fueron interpretados inicialmente por los investigadores, pero a fin de evitar sesgo de los datos se solicitó el apoyo de un experto en epidemiología, conocedor de los programas estadísticos, enriqueciendo el análisis.

Se aplicó análisis no paramétrico de Kruskall y Wallis ${ }^{3}$ que sirve para probar si un grupo de datos proviene de la misma población, realizar comparaciones múltiples entre grupos (análisis de muestras independientes provenientes de poblaciones diferentes) y para las variables cualitativas ordinales.

3 KRUSKAL, William H. and WALLIS, W. Allen. Use of ranks in one-criterion variance analysis. En: Journal of the American Statistical Association. December, 1952, 47 (260), pp. 583-621. 
Se decidió que este análisis era el más adecuado, teniendo en cuenta que la muestra definida para el estudio no se podía establecer desde un principio, sino que estaba sujeta al número de líderes con los que contara cada organización, pero sí era importante que participaran el 100\% de los líderes, de ahí que se solicitaran las respectivas constancias.

\section{RESULTADOS}

\section{Resultados cualitativos}

Se efectuaron los grupos focales que contaron con los siguientes miembros de los equipos organizacionales:

- Organización 1: entidad acreditada: 10 miembros

- Organización 2: entidad habilitada (organización control): 6 miembros
- Organización 3: entidad certificada: 11 miembros

Las entrevistas se grabaron en medio electrónico y se transcribieron para ser incluidas en el software de análisis cualitativo ATLAS. ti 6.2

Como resultado del grupo focal surgieron nuevas categorías, las cuales denominamos "emergentes".

El documento primario surge como resultado de la inclusión de las entrevistas en el software ATLAS.ti cuyo aporte radica en la organización de las citas, lo cual permite definir las categorías emergentes que a su vez son codificadas para definir el grupo de familias. En la figura 1 se representan las etapas descritas anteriormente.

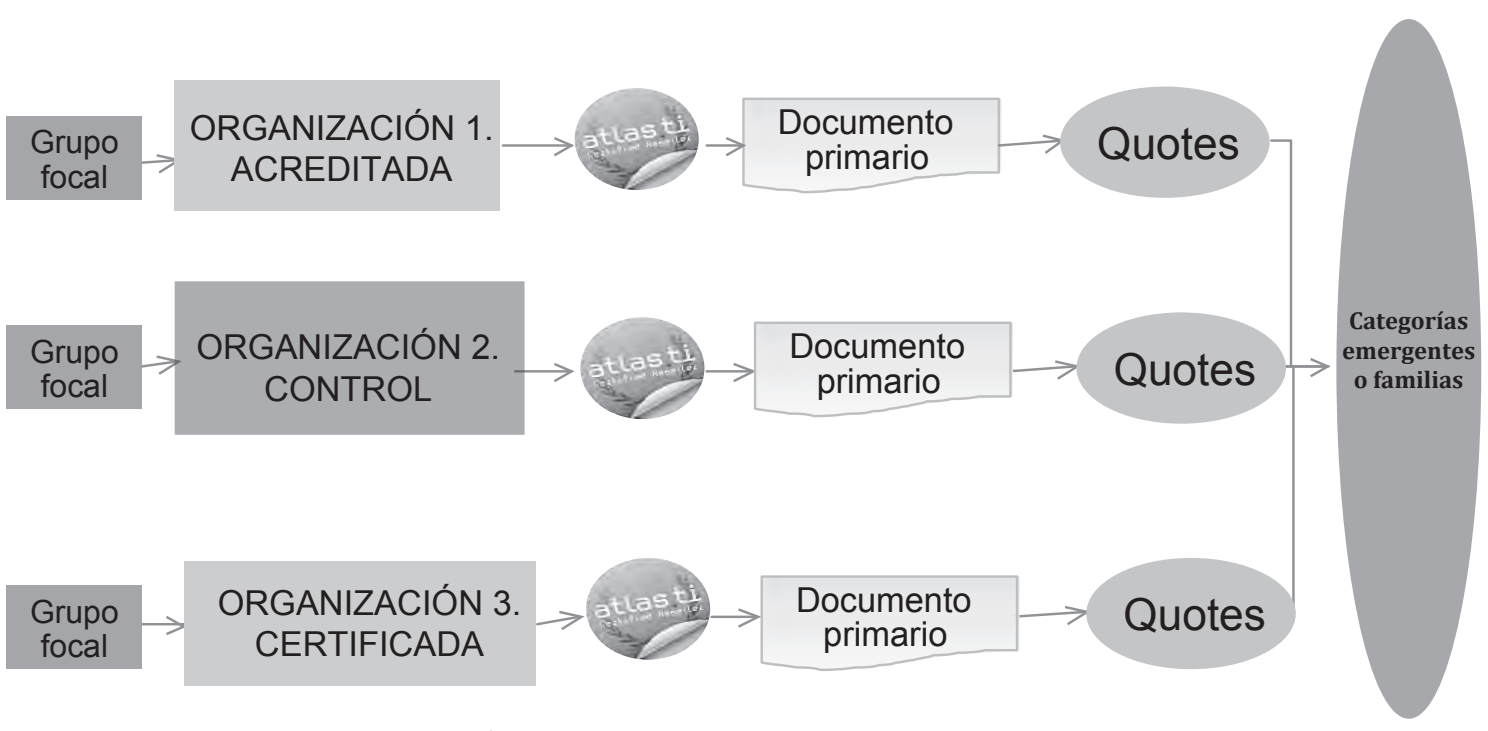

Figura 1. Esquema de obtención de categorías emergentes.

De esta manera, las categorías emergentes resultado del procesamiento y análisis de los documentos primarios junto con las variables que la conforman, sirvieron de marco para la preparación del instrumento de recolección de información. 
Los hallazgos anteriores se contrastaron con las categorías a priori surgidas del análisis del marco teórico y se soportan en la bibliografía de la investigación con respecto a la pregunta de investigación: ¿¿Cuál es la relación entre la gestión de calidad y el reporte de la ocurrencia de eventos adversos en la atención del paciente en dos instituciones de salud de Bogotá que han implementado sistemas de gestión de calidad reconocidos?

De las cinco categorías emergentes se tomaron tres en consideración a su afinidad con el problema de investigación y por los resultados cuantitativos obtenidos en las instituciones participantes, sin restar importancia a las dos categorías no incluidas, las cuales pueden considerarse para estudios posteriores teniendo en cuenta que son bastante amplias para generar una nueva investigación.

Las categorías seleccionadas fueron:

- Reporte del evento adverso e incidente

- Mejora al sistema

- Cultura del reporte

Categorias excluidas:

- Cultura de la culpa y el silencio

- Seguridad del paciente

\section{Hallazgos de los grupos focales por categoría emergente}

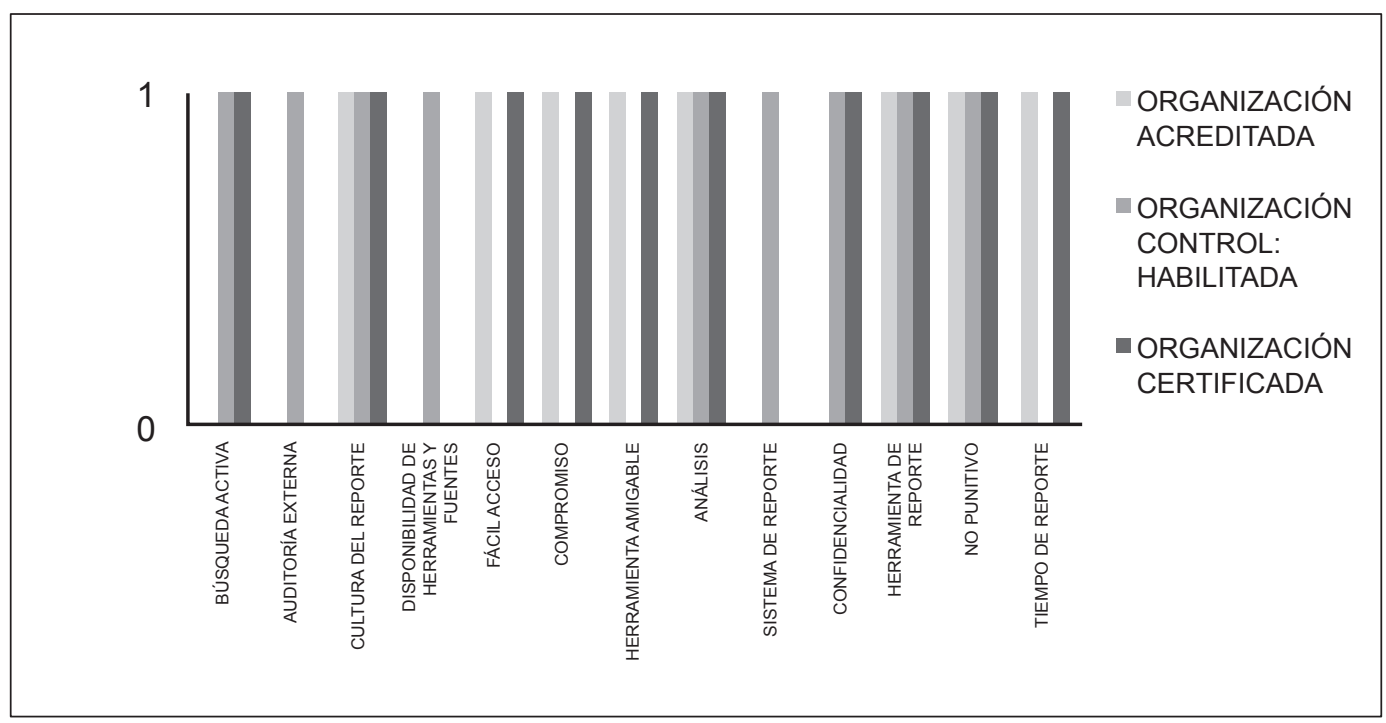

Fiǵura 2. Variables categoría reporte del evento adverso e incidentes.

Inferencias de los análisis e impacto de las variables de la categoría Reporte del Evento Adverso e Incidentes y Análisis Categorial

Para esta categoría, según la figura 2, la organización de control tiene presencia en las variables: Búsqueda
Activa, Auditoría Externa, Cultura del Reporte, Disponibilidad de Herramientas, Análisis, Sistema de Reporte, Confidencialidad, Herramienta del Reporte y No Punitivo; dado que las tres organizaciones que están habilitadas deberían estar en igualdad de condiciones respecto a estas variables; sin embargo, se 
encontró que no se hizo mención en la organización acreditada y certificada para las variables: Auditoría Externa, Disponibilidad de Herramientas y Sistema de Reporte. De este hallazgo se puede inferir que estos elementos pueden potencializar el desempeño del sistema de gestión de calidad con el cual cuentan.

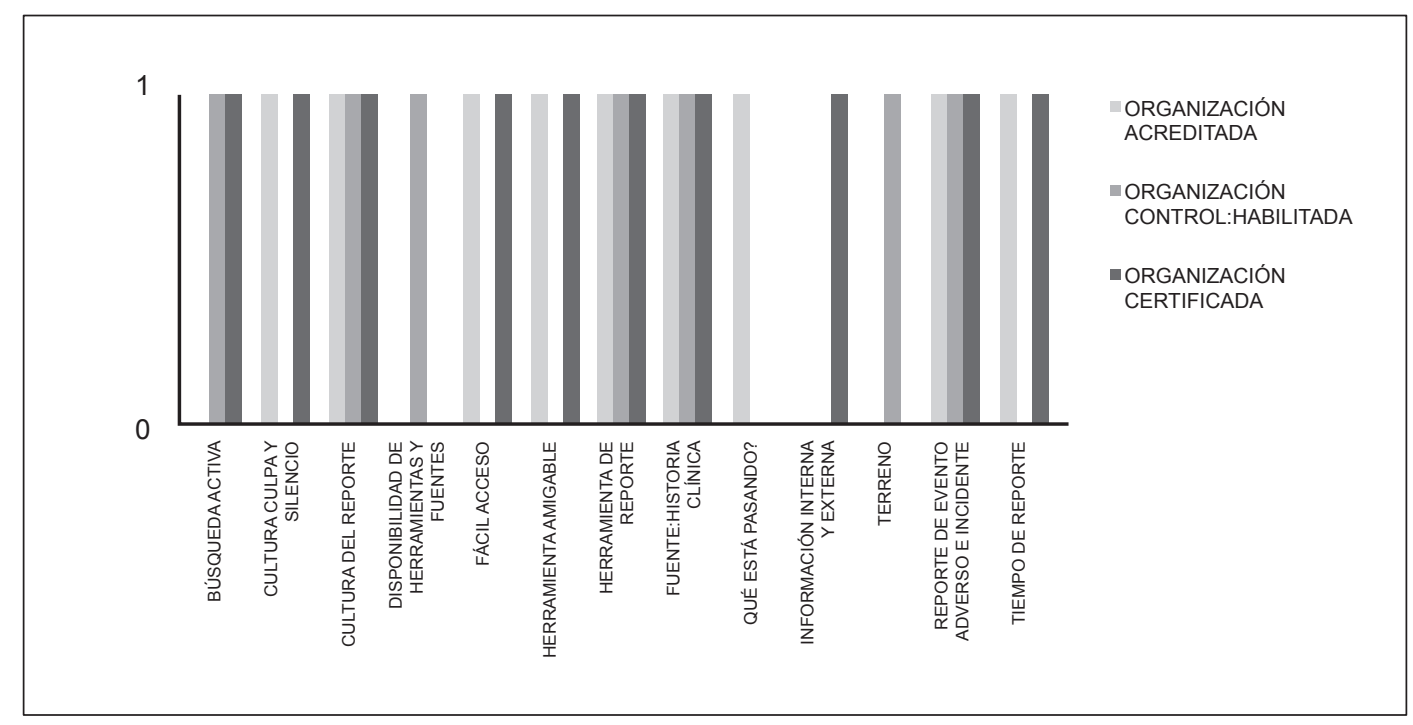

Figura 3. Variables categoría cultura del reporte.

Inferencias de los análisis e impacto de las variables de la categoría Cultura del Reporte y Análisis Categorial.

De acuerdo con la figura 3 , esta categoría se caracteriza por tener variables presentes en las organizaciones acreditadas y certificadas y ausentes en la organización de control; de esta manera las variables: Cultura Culpa y Silencio, Fácil Acceso, Herramienta Amigable y Tiempo de Reporte se constituyen en un aporte que los sistemas implementados, tanto en la organización Acreditada como en la Certificada, hacen para fomentar una cultura del reporte, es decir, para consignar por escrito los eventos sucedidos.
Es importante entonces destacar el aporte de la norma en su aplicación a nivel documental como apoyo a los aspectos relacionados con la seguridad del paciente.

Por otro lado, se evidencia la presencia de las variables: Cultura del Reporte, Herramienta de Reporte, Fuente: Historia Clínica y Reporte de Evento Adverso, en las tres organizaciones, indicando la obligatoriedad de la presencia de estas variables en una organización habilitada (de control) tenga o no otro sistema implementado para esta categoría, teniendo en cuenta que todas las organizaciones de salud deben efectuar el seguimiento al reporte de los eventos adversos desde la resolución 1446 del 2006. 


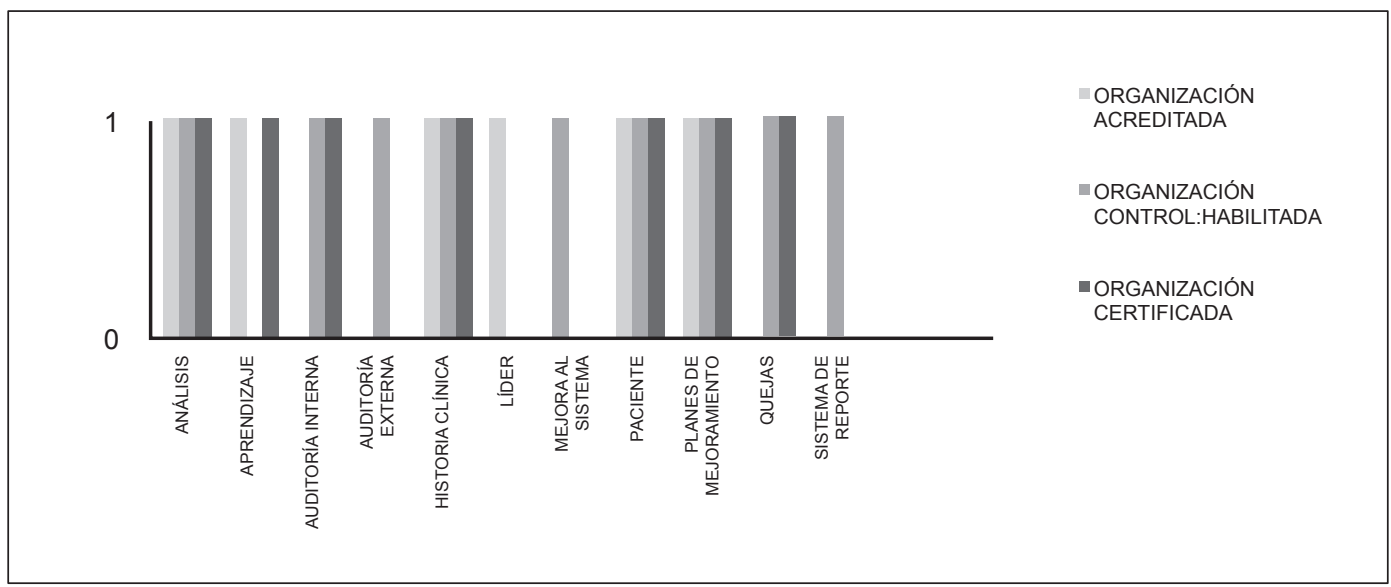

Figura 4. Variables categoría mejora al sistema.

Inferencias de los análisis e impacto de las variables de la categoría Mejora al Sistema y Análisis Categorial:

L figura 4 muestra que las variables Análisis, Historia Clínica, Paciente y Planes de Mejoramiento se encuentran presentes en las tres organizaciones, tanto en la Acreditada y Certificada como en la de Control. Este comportamiento permite concluir que para la mejora al sistema de cada organización estas variables deben estar presentes como resultado de la implementación del sistema obligatorio.

Es importante resaltar que el aprendizaje fue la única variable presentada en las organizaciones Certificada y Acreditada y no en la organización de Control, siendo esto un aspecto diferenciador al implementar uno de estos sistemas en pro al mejoramiento del sistema.

Las variables Auditoría Interna y Quejas solo se encuentran presentes en la organización de Control y en la organización Certificada, y estas no fueron mencionadas en la organización Acreditada pero por otro lado, solo la organización Acreditada considera variables importantes como la presencia de un líder y mejora al sistema dando un valor agregado a la categoría Mejora al Sistema.

\section{Resultados cuantitativos}

Se diseñó y aplicó una encuesta con 27 preguntas, con cuatro categorías de variables para evaluar el grado de implementación del sistema de reporte de eventos adversos en tres organizaciones, dentro de las que se encontraban una organización acreditada, una organización certificada y una de control con el sistema único de habilitación que se denominaron así:

- Acreditada: organización 1

- Habilitada de Control: organización 2

- Certificada: organización 3 


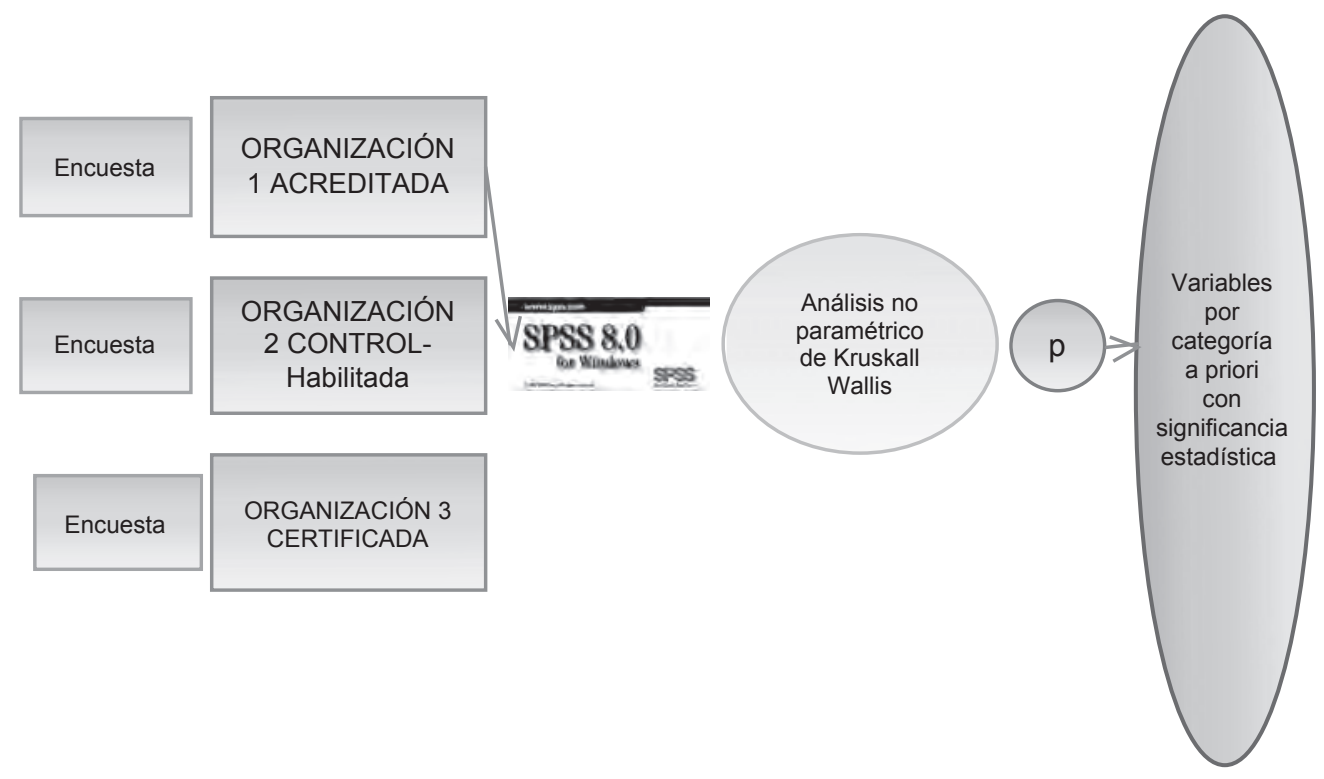

Figura 5. Esquema de obtención de variables por categoría.

Al comparar el grado de implementación del sistema de reporte en las tres organizaciones, de acuerdo con las cuatro categorías a priori definidas, se obtuvieron los siguientes resultados al ser procesados en el software SPSS versión 8.0 y a través del análisis no paramétrico de Kruskall Wallis se obtuvo el valor de $p$ (nivel de significancia estadística):

\section{Inferencias de los análisis e impacto cuantitativo}

\section{A. Categoría de Reporte del Evento Adverso}

Al realizar la comparación entre las tres organizaciones con respecto a esta categoría, y de acuerdo con los datos obtenidos, los resultados de la organización 3, Certificada, no son estadísticamente significativos y se identifican debilidades en las variables sistema de notificación del evento adverso, sistema de reporte de eventos adversos para usuarios y familias, revisiones sistemáticas al sistema de notificación y mecanismos de reporte y en retroalimentación de los análisis de los eventos adversos; la organización 1, Acreditada, cumple con las variables, excepto con el sistema de reporte de eventos adversos para usuarios y familias; la organización 2, Control cumple con las variables de esta categoría.

\section{B. Categoría de Cultura de la Seguridad}

En la organización 3, Certificada, para esta categoría los resultados no son estadísticamente significativos y se encuentran debilidades en las variables: Campañas de Promoción del Reporte del Evento Adverso, Mejora de la Cultura de Seguridad por Reporte de Evento Adverso, Control del Evento Adverso por Análisis y Seguimiento, Conocimiento de la taxonomía, Comité de Análisis Capacitado, Tiempo de Reporte de Evento Adverso y Tiempo de Análisis; para la organización 1, Acreditada, se cumple con todas las variables excepto con la Promoción del Reporte de Eventos Adversos con Incentivos; en la organización 2, de Control, hay debilidad en inducción y reinducción en Temáticas de Reporte de Evento Adverso y Promoción del Reporte del Evento Adverso con Incentivos.

\section{C.Categoría Gestión de la Calidad}

En la organización 3, Certificada, se obtuvo diferencia significativa para esta categoría en las variables: 
Reporte de Evento Adverso permite aprendizaje y mejora de la calidad, cambio en el comportamiento en la institución por el reporte, Identificación de Riesgos que conducen a la mejora y cambio en los procesos y sistema por el reporte de evento adverso. Aunque la organización 3 está certificada, los participantes manifiestan que, de manera no satisfactoria o de manera aceptable, se cumple con las variables Reporte de Evento Adverso, permite aprendizaje y mejora de la calidad, cambio en los procesos y sistema por el reporte de evento adverso y cambio en el comportamiento en la institución por el reporte; las organizaciones 1 y 2 si cumplen con las variables de esta categoría.

\section{Categoría de la Cultura de la Culpa y el Silencio}

En esta categoría las organizaciones 1 y 2 cumplen en todas las variables establecidas. En la organización 3 los participantes perciben que no hay acciones para acabar con Cultura de la Culpa, Promover Cultura del Reporte.

\section{DISCUSIÓN}

\section{Inferencias de los análisis e impactos cualitativos y cuantitativos}

$\mathrm{Al}$ obtener los datos cualitativos de cada una de las organizaciones son enriquecidos con los datos cuantitativos de manera que se obtengan inferencias completas con respecto a las categorías planteadas, desde las perspectivas y apreciaciones de los participantes. A continuación se sintetizan las inferencias:

Tabla 1. Categorías

\begin{tabular}{|c|c|c|}
\hline \multirow{2}{*}{\multicolumn{3}{|c|}{$\begin{array}{l}\text { CATEGORÍA CUANTITATIVA: REPORTE DEL EVENTO ADVERSO } \\
\text { CATECORÍ }\end{array}$}} \\
\hline CATEGORÍA CUANTIT & & VERSO E INCIDENTE \\
\hline $\begin{array}{l}\text { ORGANIZACIÓN } 1 \\
\text { ACREDITADA }\end{array}$ & $\begin{array}{c}\text { ORGANIZACIÓN } 2 \\
\text { HABILITADA(CONTROL) }\end{array}$ & $\begin{array}{l}\text { ORGANIZACIÓN } 3 \\
\text { CERTIFICADA }\end{array}$ \\
\hline $\begin{array}{l}\text { Debilidades en cuanto al sistema de reporte de } \\
\text { los eventos adversos para usuarios y familia. }\end{array}$ & $\begin{array}{l}\text { Cumple con todas las variables de la } \\
\text { categoría cuantitativa del reporte del evento } \\
\text { adverso. }\end{array}$ & $\begin{array}{l}\text { * Debilidades en el sistema de notificación de } \\
\text { los eventos adversos, sistema de reporte del } \\
\text { evento adverso para usuarios y familia, revi- } \\
\text { sión al sistema de notificación, mecanismos } \\
\text { de reporte y retroalimentación de los análisis. }\end{array}$ \\
\hline $\begin{array}{l}\text { * Cuenta con el compromiso de la Dirección } \\
\text { General. } \\
\text { * Cuenta con líder para el Comité de Análisis de } \\
\text { Eventos Adversos e Incidentes. } \\
\text { * Realiza análisis de causa raíz, los cuales son } \\
\text { socializados con la organización. } \\
\text { * Cuenta con sistema de reporte en la intranet } \\
\text { con taxonomía definida. } \\
\text { * Se da a conocer a los colaboradores en los proce- } \\
\text { sos de inducción y capacitación, promoviendo } \\
\text { la confianza hacia el reporte y la no punición. } \\
\text { * Tiene definida una estrategia de búsqueda de } \\
\text { incidentes a través de ronda diaria con retroali- } \\
\text { mentación inmediata. } \\
\text { * Tiene definidos los tiempos de reporte y de } \\
\text { análisis. }\end{array}$ & $\begin{array}{l}\text { * Implementación del sistema de reporte } \\
\text { sin censurar y descalificar al personal. } \\
\text { * Cuenta con un protocolo de análisis ante } \\
\text { el reporte de eventos adversos, se levanta } \\
\text { plan de mejoramiento y se socializa. } \\
\text { * Se apoya en fuentes de información para } \\
\text { la detección de eventos adversos en las } \\
\text { auditorías interna y externa, quejas de } \\
\text { los usuarios y los registros en historias } \\
\text { clínicas. } \\
\text { * La organización propone incentivar el } \\
\text { auto reporte, incentivar a las personas } \\
\text { que generen el reporte. } \\
\text { * La organización cuenta con un formato } \\
\text { en físico para el sistema de reporte. } \\
\text { * Tiene identificado los riesgos y eventos } \\
\text { adversos por procesos. }\end{array}$ & $\begin{array}{l}\text { * Los colaboradores manifiestan temor hacia } \\
\text { el reporte, piensan que es punitivo y puede } \\
\text { llevar a procesos disciplinarios y terminación } \\
\text { de contratos. } \\
\text { * Proponen que se debe fortalecer la inducción } \\
\text { y reinducción al personal, la retroalimenta- } \\
\text { ción, el uso de la intranet como mecanismo } \\
\text { para implementar el sistema de reporte. } \\
\text { * Para la gestión del sistema de reporte se } \\
\text { cuenta con un líder que conoce y maneja el } \\
\text { formato de reporte, pero no es conocido por } \\
\text { todos los miembros de la organización. } \\
\text { * Proponen que el sistema de reporte debe } \\
\text { enriquecerse por medio de formatos, análisis, } \\
\text { promover el autocontrol, incentivos y buena } \\
\text { comunicación para crear cultura del reporte. }\end{array}$ \\
\hline
\end{tabular}




\begin{tabular}{|c|c|c|}
\hline \multicolumn{3}{|c|}{ CATEGORÍA CUANTITATIVA: CULTURA DE LA SEGURIDAD } \\
\hline \multicolumn{3}{|c|}{ CATEGORÍA CUANTITATIVA:CULTURA DE LA CULPA Y DEL SILENCIO } \\
\hline \multicolumn{3}{|c|}{ CATEGORÍA CUALITATIVA: CULTURA DEL REPORTE } \\
\hline $\begin{array}{l}\text { ORGANIZACIÓN } 1 \\
\text { ACREDITADA }\end{array}$ & $\begin{array}{c}\text { ORGANIZACIÓN } 2 \\
\text { HABILITADA(CONTROL) }\end{array}$ & $\begin{array}{c}\text { ORGANIZACIÓN } 3 \\
\text { CERTIFICADA }\end{array}$ \\
\hline $\begin{array}{l}\text { Debilidades en la promoción del reporte del evento } \\
\text { adverso con incentivos }\end{array}$ & $\begin{array}{l}\text { *Debilidades en la inducción y reinducción } \\
\text { en temáticas de reporte del evento adverso y } \\
\text { promoción del reporte del evento adverso con } \\
\text { incentivos. }\end{array}$ & $\begin{array}{l}\text { *Debilidades hacia la campaña de } \\
\text { promoción del reporte del evento } \\
\text { adverso, mejora de la cultura por el } \\
\text { reporte del evento adverso, análisis } \\
\text { y seguimiento al evento adverso, } \\
\text { conocimiento a la taxonomía y capa- } \\
\text { citación a los integrantes del Comité } \\
\text { de Análisis. }\end{array}$ \\
\hline $\begin{array}{l}\text { Cumple con las variables de la categoría de la culpa } \\
\text { y el silencio }\end{array}$ & $\begin{array}{l}\text { Cumple con las variables de la categoría de la } \\
\text { culpa y el silencio }\end{array}$ & $\begin{array}{l}\text { Los participantes manifiestan que no } \\
\text { hay acciones para acabar con la cultura } \\
\text { de la culpa y promover la cultura del } \\
\text { reporte. }\end{array}$ \\
\hline $\begin{array}{l}\text { *Los participantes consideran que el liderazgo y el } \\
\text { compromiso hacia la promoción de la cultura del } \\
\text { reporte debe partir de la dirección general, con polí- } \\
\text { ticas institucionales y el fortalecimiento a través de la } \\
\text { educación de los colaboradores y de los usuarios. } \\
\text { *Enriquece la cultura a través de la referenciación } \\
\text { con otras entidades del mismo nivel de complejidad. } \\
\text { *Cuenta con sistema de reporte en la intranet de fácil } \\
\text { acceso, amigable y conocido por toda la organización. } \\
\text { *Cuenta con Comité de Análisis de reporte de inci- } \\
\text { dentes y eventos adversos, apoyado por un líder. } \\
\text { *Consideran que la sensibilización y los incentivos } \\
\text { son estrategias claves para motivar hacia la cultura } \\
\text { del reporte. } \\
\text { *Parten de la habilitación, definiendo estrategias para } \\
\text { minimizar el riesgo. }\end{array}$ & $\begin{array}{l}\text { *La cultura del reporte debe partir desde el } \\
\text { compromiso de la Junta Directiva, promoviendo } \\
\text { la cultura del reporte a través de la sensibilización } \\
\text { y la inversión económica. } \\
\text { *Contar con recurso humano suficiente y } \\
\text { capacitado. } \\
\text { *La importancia de la dotación de equipos y } \\
\text { lo necesario para implementar el sistema de } \\
\text { notificación para toda la organización. } \\
\text { * Reconocer los lineamientos de la cadena de } \\
\text { cumplimientos desde la identificación de riesgos } \\
\text { por procesos, la capacitación, análisis, seguimien- } \\
\text { to y retroalimentación. } \\
\text { *Contar con disponibilidad y accesibilidad a los } \\
\text { sistemas de información y que haya un buen } \\
\text { manejo confidencial. } \\
\text { *Cuenta con un equipo de líderes que han inte- } \\
\text { riorizado lo relacionado con el sistema de reporte } \\
\text { y el fortalecimiento de la cultura. }\end{array}$ & $\begin{array}{l}\text { *Motivación al personal con un } \\
\text { proyecto de lanzamiento y apren- } \\
\text { dizaje organizacional, que incluya } \\
\text { capacitación en taxonomía, sistema de } \\
\text { reporte, análisis mejora y auditoría. } \\
\text { *La cultura de cada persona hace que } \\
\text { no se reporte fielmente. } \\
\text { *Promover la detección de fallas en la } \\
\text { atención de los pacientes. } \\
\text { *Enriquecer la cultura desde la } \\
\text { información interna y externa. } \\
\text { *Generar una cultura de cambio a } \\
\text { partir de las acciones preventivas y } \\
\text { correctivas generadas desde el análisis } \\
\text { de los eventos adversos. }\end{array}$ \\
\hline
\end{tabular}




\begin{tabular}{|c|c|c|}
\hline \multirow{2}{*}{\multicolumn{3}{|c|}{$\begin{array}{l}\text { CATEGORÍA CUANTITATIVA: GESTIÓN DE LA CALIDAD } \\
\text { CATEGORÍA CUANTITATIVA:MEJORA AL SISTEMA }\end{array}$}} \\
\hline & & \\
\hline $\begin{array}{l}\text { ORGANIZACIÓN } 1 \\
\text { ACREDITADA }\end{array}$ & $\begin{array}{c}\text { ORGANIZACIÓN } 2 \\
\text { HABILITADA(CONTROL) }\end{array}$ & $\begin{array}{l}\text { ORGANIZACIÓN } 3 \\
\text { CERTIFICADA }\end{array}$ \\
\hline $\begin{array}{l}\text { Cumple con las variables establecidas para } \\
\text { esta categoría. }\end{array}$ & $\begin{array}{l}\text { Cumple con las variables establecidas para esta } \\
\text { categoría. }\end{array}$ & $\begin{array}{l}\text { *Debilidades hacia la campaña de promoción del } \\
\text { reporte del evento adverso, mejora de la cultura } \\
\text { por el reporte del evento adverso, análisis y } \\
\text { seguimiento al evento adverso, conocimiento a } \\
\text { la taxonomía y capacitación a los integrantes del } \\
\text { Comité de Análisis. }\end{array}$ \\
\hline $\begin{array}{l}\text { *La Organización realiza análisis causal con } \\
\text { el fin de generar planes de mejoramiento } \\
\text { que minimicen el riesgo y se apliquen } \\
\text { acciones que se conviertan en aprendi- } \\
\text { zaje organizacional y mejora del sistema, } \\
\text { contando con el apoyo de un líder. } \\
\text { *Las acciones de mejora son enfocadas a } \\
\text { la satisfacción del usuario y su familia, lo } \\
\text { involucra en los procesos educativos en la } \\
\text { organización. } \\
\text { Desde las fuentes de información, la } \\
\text { historia clínica, busca la estandarización y la } \\
\text { exploración del evento adverso. } \\
\text { *A través de relaciones con otras organiza- } \\
\text { ciones que tienen experiencias exitosas, se } \\
\text { mejoran procesos en la organización. }\end{array}$ & $\begin{array}{l}\text { *Desde las fuentes de información: auditorías } \\
\text { internas y externas aportan elementos para } \\
\text { realizar análisis causal y acciones de mejora con } \\
\text { el fin de evidenciar el mejoramiento continuo y } \\
\text { que impacte de manera positiva en los usuarios } \\
\text { y en el cliente interno. } \\
\text { *El sistema de reporte que incluya la gestión } \\
\text { del evento adverso resulta efectivo en la } \\
\text { organización pero requiere el compromiso y } \\
\text { la motivación hacia el reporte por parte de los } \\
\text { colaboradores. } \\
\text { *Fuentes de información: las quejas aportan y } \\
\text { enriquecen el sistema de reporte y además es } \\
\text { evidencia de la escucha al usuario por parte de } \\
\text { la organización para gestionar sus necesidades y } \\
\text { expectativas. }\end{array}$ & $\begin{array}{l}\text { *Se cuenta con líder responsable del reporte del } \\
\text { evento adverso y el diligenciamiento del formato } \\
\text { en físico. } \\
\text { *Se gestiona el análisis del evento adverso y se } \\
\text { aplican las acciones correctivas y preventivas, } \\
\text { según el caso. } \\
\text { *La organización reconoce la importancia de } \\
\text { contar con personal capacitado en todo lo rela- } \\
\text { cionado con el evento adverso y además propone } \\
\text { mecanismos para realizar autocapacitación. } \\
\text { *Fortalecer los análisis causales con las acciones } \\
\text { correctivas y preventivas, que evidencien la } \\
\text { mejora al sistema. } \\
\text { *A través del Sistema de Auditoría, identificar } \\
\text { oportunidades de mejora. } \\
\text { *Tener en cuenta las quejas verbales de los } \\
\text { usuarios dentro de los procesos de atención, ge- } \\
\text { nerando mecanismos de captura de la información } \\
\text { y aplicando acciones correctivas o preventivas, } \\
\text { según el caso. }\end{array}$ \\
\hline
\end{tabular}

\section{CONCLUSIONES}

Los anteriores resultados muestran como respuesta al problema planteado, las relaciones y el comportamiento en las diferentes organizaciones con respecto a los sistemas de gestión de calidad y al reporte de los eventos adversos, evidenciando que sistemas de calidad como la Acreditación articulado a la NTC ISO 9001:2008 brindan resultados satisfactorios en cuanto a que se percibe la cultura organizacional hacia la calidad y el mantenimiento de la misma al interior de la institución. Esto sirve de modelo a las organizaciones de salud, de tal manera que los sistemas se articulen y sean un apoyo entre sí y no que se manejen de forma independiente.
Cabe anotar que los resultados encontrados en la entidad certificada reflejan un trabajo no articulado, donde todavía se perciben falencias a nivel de cultura, capacitación y no hay trabajo en equipo. Aunque se da cumplimiento a los requisitos de la NTC ISO 9001:2008, la articulación con los otros sistemas es aún deficiente.

El comportamiento de la organización habilitada es destacable, pues desde los sistemas obligatorios ha logrado articulación y muestra avances significativos hacia la cultura de seguridad y específicamente en el desarrollo de la gestión del reporte de los eventos adversos. 


\section{Organización Acreditada}

- Cuenta con los sistemas de Habilitación y NTCISO 9001:2008, tiene establecido un sistema de notificación que es conocido por toda la organización, es amigable y sencillo, lo cual permite un fácil acceso, disponible para el 100\% de los colaboradores, pero para llevarlo a cabo ha implementado un programa de capacitación en sistema de notificación, taxonomía y sensibilización hacia su uso de forma voluntaria y confidencial, que se fortalece desde los procesos de inducción y reinducción. Tiene establecido que desde el proceso de calidad y seguridad del paciente se realizan revisiones sistemáticas al sistema de notificación. En esta organización se destaca el proceso de retroalimentación sobre los resultados de los análisis de los eventos adversos, contando con un líder específico de seguridad del paciente, quien despliega la información hacia los líderes de los procesos y es como la acreditación aporta a la organización con el desarrollo y el fomento del trabajo en equipo.

\section{Organización Habilitada (Control)}

- Desde los estándares del Sistema Obligatorio de Garantía de la Calidad, Habilitación, Sistema de Información y los acercamientos al Sistema Único de Acreditación, enfatiza en la importancia de promover la cultura del reporte, con su respectivo sistema, el cual se encuentra en la intranet, cuenta con un líder, utiliza varias fuentes de información, entre ellas, los aportes desde la historia clínica, auditorías internas y encuestas de los usuarios. Propone motivar hacia el autorreporte y recalca la importancia de documentar a través de protocolos y la necesidad de realizar el análisis de los eventos llevándolo a planes de mejoramiento, que se socializan y no generan acciones de censura y acciones punitivas.

\section{Organización Certificada}

- Se observa desarticulación entre el Sistema de Gestión de la Calidad y los otros sistemas obligatorios, e igualmente con respecto al reporte de la ocurrencia de los eventos adversos, sin embargo, la organización cuenta con un sistema de notificación, capacita en sistema de notificación y reporte del evento adverso promoviendo que sea de manera confidencial y voluntario.

- En esta organización, aunque cuenta con la certificación, la categoría de Cultura de la Seguridad es débil, lo cual requiere un replanteamiento y articulación de manera que desde los requisitos de la norma se dé cumplimiento a toda la gestión de las variables planteadas para esta categoría, aunque se evidencian algunas fortalezas a nivel de capacitación sobre reporte de evento adverso, inducción y reinducción, Comité de Análisis y retroalimentación del evento adverso, métodos de aprendizaje desde el reporte y fuentes alternativas de información.

- En la categoría de Gestión de la Calidad, la organización no percibe desde el reporte del evento adverso, el aprendizaje y la mejora de la calidad, cambio en los procesos y sistema, cambio en el comportamiento en la institución. Coinciden en que la gestión del riesgo si ha sido un aporte que ha conducido a la mejora.

- La categoría de la Cultura de la Culpa y el Silencio, se presenta en las organizaciones como la oportunidad de reducir el temor hacia el reporte punitivo, fomentar la cultura del reporte, mantener un sistema de reporte independiente de cualquier cargo de poder para ejercer castigo y acabar con la cultura de la culpa. En la organización acreditada y habilitada, los participantes lo perciben en el interior de sus organizaciones a diferencia de la certificada 
que considera que no se emprenden acciones para promover la cultura del reporte y acabar con la cultura de la culpa.

- La organización certificada independiente al establecimiento del sistema de gestión, evidencia problemáticas en la duplicación de funciones, reprocesos y falta de directrices claras, lo cual está impactando de manera negativa la gestión de la organización.

- Es importante resaltar que el verdadero propósito de contar con un sistema de gestión de calidad reconocido, no radica en la obtención del certificado, sino en la generación de beneficios a un usuario que requiere el máximo nivel de cuidados, derivada de la necesidad de una atención en salud.

\section{RECOMENDACIONES}

Las entidades de salud que deseen implementar sistemas de gestión deben realizar un paralelo entre los mismos para identificar los puntos comunes y permitir la integración, de tal forma que se nutran y se pueda percibir la relación entre ellos.

Fortalecer el sistema de reporte de eventos adversos para los usuarios y sus familias, teniendo en cuenta que en uno de los principios orientadores de la Política de Seguridad del Paciente del Ministerio de la Protección Social recalca la importancia de la alianza con el paciente y su familia.

Promover la revisión sistemática al sistema de notificación, definiendo un responsable para ello y que a su vez realice la retroalimentación del análisis de los eventos adversos a los líderes definidos para los procesos $\mathrm{y}$, adicionalmente, capacite a sus colaboradores en mecanismos de reporte.
Promover el reporte de los eventos adversos a través de incentivos, que den confianza a los colaboradores y se minimice la cultura del silencio en el interior de los procesos asistenciales.

Fortalecer los programas de inducción y reinducción en temáticas relacionadas con seguridad del paciente.

Definir, desde el planteamiento inicial de la implementación del Sistema de Gestión de la Calidad, las contribuciones que puede hacer hacia el reporte de los eventos adversos y ser desplegado a toda la organización.

Crear estrategias que promuevan el reporte del evento adverso en el interior de las organizaciones, con el enfoque de acabar con la culpa y el silencio y que sea una directriz desde la alta dirección libre de castigo.

\section{BIBLIOGRAFÍA}

AHRQ. Medical Errors: The Scoop of the problem. Agency for Health Research and Quality. Estados Unidos de Norteamérica. February, 2000.

BRENNAN N., Leape LL. Incidence of adverse events and negligencein hospitalized patients. En: N Engl J Med, 1991, 324, pp. 370-376.

BRUUN JENSEN, Casper. Sociology, systems and (patient) safety: knowledge translations in healthcare policy. En: Sociology of Health \& Illness, 2008, Vol. 30 No. 2, pp. 309-324.

CAJIGAS, Blanca. La Política de Calidad en Salud en Colombia: En: Presentación del Ministerio de la Protección Social, noviembre, 2007. 
DONABEDIAN, A. Aspects of medical care administration: specifying requirements for health care. Massachusetts: Harvard University Press, 1973.

FRANCO, Astolfo. La seguridad clínica de los pacientes: entendiendo el problema. En: Colombia Médica, abril-junio, 2005, Vol. 36 No. 2.

GIRALDO, Marcela. Proyecto ibeas y la políica de seguridad del paciente en Colombia. Ministerio de Salud y la Protección Social.

HERNÁNDEZ SAMPIERI, Roberto. Metodología de la investigación. Madrid, España: Ed. McGraw Hill, 2010.

KRUSKAL, William H. and WALLIS, W. Allen. Use of ranks in one-criterion variance analysis. En: Journal of the American Statistical Association. December, 1952, 47 (260), pp. 583-621.
MINISTERIO DE SALUD Y PROTECCIÓN SOCIAL. Lineamientos para la implementación de la Política de Seguridad del Paciente. Noviembre, 2008.

ORGANIZACIÓN MUNDIAL DE LA SALUD. Informe de la $55^{a}$ Asamblea Mundial. Ginebra: OMS, marzo, 2002.

RAMOS DBN. El Control de calidad de la atención de salud. La Habana: Ed. Ciencias Médicas, 2004, pp. 22-39.

WORLD HEALTH ORGANIZATION. World Alliance for Patient Safety: Forward programme. Geneva: WHO Library, october, 2004. 